\title{
Delirium in hospitalized patients: Cohort observational in infirmary of medical clinic
}

\author{
Rilva Lopes de Sousa-Muñoz ${ }^{1^{*}}$, Leila Coutinho Taguchi ${ }^{1,2}$, Mara Rufino de Andrade $^{1}$, \\ Katyara Mylena Silveira Ribeiro Lima ${ }^{1}$ \\ ${ }^{1}$ Federal University of Paraiba (UFPB), João Pessoa, Brazil; ${ }^{*}$ Corresponding Author: rilva@ccm.ufpb.br \\ ${ }^{2}$ Lauro Wanderley University Hospital (LWUH), João Pessoa, Brazil
}

Received 17 January 2012; revised 18 February 2012; accepted 12 March 2012

\begin{abstract}
Objectives: To determine prevalence, incidence and risk factors of delirium in hospitalized patients in the infirmary of medical clinic of the Lauro Wanderley University Hospital. Method: An observational cohort study evaluating 140 patients over 18 years between admission and discharge. The survey instrument was the Confusion Assessment Method. Results: The prevalence of delirium was $5.7 \%(8 / 140)$ and the incidence, $2.1 \%$. In patients with delirium were observed alteration of sleep-wake cycle (6/75\%), altered level of consciousness (6/75\%), disorganized thinking $(5 / 62,5 \%)$ and psychomotor retardation (5/62 5\%). There was a significant association of delirium and age, mechanical restraint in bed, prior history of cognitive impairment, auditory and visual sensory deficit, psychiatric hospitalization, poor health status and mortality. Conclusions: The prevalence of delirium at admission was lower than that found in other studies, but it was a predictor factor of hospital mortality.
\end{abstract}

Keywords: Delirium; Hospitalization; Risk Factors; Mortality

\section{INTRODUCTION}

Delirium is a disorder located on the border between neuropsychiatry and other medical specialties, appearing in the course of several diseases, especially in the context of internal medicine and geriatrics [1]. This is a neuropsychiatric disorder secondary to a general medical condition. It is highly prevalent in hospitalized patients and has significant impact on morbidity and mortality [2].

It has been shown a progressive increase in the incidence of this syndrome in the population over 60 years [2], but with delayed detection in $32 \%$ - $67 \%$ of cases [3].
However, Rudberg et al. highlight the substantial clinical heterogeneity among patients who develop delirium during hospitalization, although there are few data on patients of different age groups or in relation to gender and clinical condition [4].

Patients with delirium are at increased risk of various complications such as pneumonia, decubitus ulcer and postoperative complications, and longer hospitalizations and poorer prognosis [5].

Given these data, this study aims to determine the prevalence, incidence, risk factors and outcome of patients with delirium hospitalized in the medical clinic of Lauro Wanderley University Hospital (LWUH).

\section{METHODS}

This study followed an observational model, cohort, in which the patient was followed from the time of admission, with or without detection of the episode of delirium, until his discharge.

Was also accomplished the capture of data for the period prior to admission. All patients hospitalized in the infirmary of medical clinic (IMC) of LWUH that meet the inclusion/exclusion criteria were recruited after signing the consent form.

The recruitment of patients was performed as accidental, with selection of patients consecutively admitted to the IMC of LWUH to obtain a sample size of 140 patients. This sample size was calculated to estimate the proportion of delirium on the population of interest, i.e., the frequency of the event, assuming that the true rate of this condition is approximately $10 \%$ and the difference between actual and estimated prevalence is up $5 \%$ at a significance level of $5 \%$.

Patients should have recruited up to 24 hours of hospitalization in the IMC. It was adopted the following inclusion criteria: 1) patients of both sexes admitted for medical treatment in the IMC of LWUH; 2) minimal aged of 18 years; 3) informed consent to participate in research. Were excluded patients with suspected or con- 
firmed diagnosis of dementia, as well as patients in a coma.

The research team was composed of two medical residents in medical clinic, two medical students and a physician. Before the formal start of the study a pretest was performed to make adjustments in the methodologycal procedures.

During the study period, the researchers daily reviewed the book of admissions of IMC, noting each new patient admitted and assessed the selection criteria, subjecting it to predetermined measurements; thus all patients had an initial assessment within the first 24 hours of their hospitalization. After the admission, the wards were visited three times a week by researchers at the same time of day (8:00 am) for monitoring of all patients included in the study.

The research instruments were the Confusion Assessment Method-CAM [6]. The CAM is an instrument designed to perform diagnosis of delirium [6], validated in Brazil [2], with sensitivity of $94.1 \%, 96.3 \%$ of specificity and reliability of 0.70 . The diagnosis of delirium requires necessarily the presence of items 1 and 2 of the CAM (acute onset and disorder of attention) and/or items 3 and 4 (disorganized thinking and altered level of consciousness).

Were recorded on a standardized form designed for this study different demographic and clinical variables: 1) demographic (age; sex; marital status; education level; occupation) and 2) clinical (main definitive diagnosis; history of reduced visual acuity and hearing loss, mechanical immobilization in bed; abuse or alcohol dependence; use of medicaments; head trauma; tuberculosis; smoking; drugs; history of depression; psychiatric hospitalization; chronic cognitive impairment, duration of hospital stay, number of medical visits and hospitalizations prior; hospital mortality).

The main definitive diagnosis responsible for hospitalization of patients in HULW was categorized by group of diseases according to the 10th International Statistical Classification of Diseases and Related Health Problems (CID-10) [7]. Immobilization was defined as mechanical restraint in bed by use of metal bars or ties to stand the patient still. History of chronic cognitive impairment was defined as the reference of the patient, family, or both, from a deficit of cognitive functions of at least six months, enough to affect the patient's ability to recognize people, perform activities of daily living or walk without help.

Data were entered, processed and analyzed using SPSS 17.0 for Windows. For the inferential analysis of the data, were stratified the different variables and used the Student t test to compare mean age between groups of patients with and without delirium, the chi-square test (or Fisher exact test) for comparison of qualitative variables, given a statistically significant difference at $p=$
0.05 .

This research project was approved by the Research Ethics Committee of LWUH (REC/LWUH), with registration SISNEP-CAAE 0070.0.126.000-07. Eligible patients were invited to participate in the research, either directly or through its caregivers, and signed a consent form.

\section{RESULTS}

The study sample consisted of 140 patients. Most patients were female (55\%). The age of the patients interviewed varied between 18 and 89 years, mean 47.3 ( \pm 17.3); 19.6\% were aged between 18 and 29 years; $36.2 \%$ between 30 and 49 years; 33.3\% between 50 and 69 years; and $10.9 \%$ over 70 years. The elderly accounted for $26.4 \%$ of the sample.

The average educational level of the patients included in the sample was $5.3( \pm 4.1)$ years. Most patients were brown $(52.9 \%)$. It was observed that $56.4 \%$ of the patients were residents in João Pessoa and the metropolitan area. The most common occupation was farming (17.1\%), followed by a stay-at-home (15.7\%), 52.9\% were active working.

It was observed that diseases of the digestive tract were the most frequent (24.3\% of diagnoses). The second category of most frequent diagnosis was cardiovascular disease (14.3\%) and rheumatic diseases (13.6\%). Lliver cirrhosis and portal hypertension related to schistosomiasis were the most frequent diseases (16.3\%), followed by the diagnosis of congestive heart failure (8.5\%) and systemic lupus erythematosus (5\%).

The prevalence of delirium at hospital admission was $5.7 \%$ (8/140), i.e., eight patients reached the cut-off point in the CAM. These eight patients in delirium presented with acute onset of this condition and disorder of attention. Most had abnormal sleep-wake cycle (6/75\%), altered level of consciousness (6/75\%), disorganized thinking (5/62, 5\%), and psychomotor retardation (5/62, $5 \%)$.

As for the outcome of patients who developed delirium or not, during follow-up, $32.1 \%$ showed a change in any item of CAM, the item 10 (change in sleep-wake cycle) suffered the most changes (22.1\%).

In the total sample $(n=140)$, through the application of CAM, were detected, as most common changes, difficulty focusing attention, altered level of consciousness and memory (Table 1), but only 5.7\% were classified as having delirium at admission.

During the hospitalization, we observed that 45 patients $(32.1 \%)$ showed variations in the score of CAM in relation to assessment of admission, while 11 patients (7.9\%) showed changes in the scale cut-off. Three patients (2.1\%) developed signs of delirium during hospi- 
Table 1. Results of applying Confusion Assessment Method (CAM) to the sample of patients hospitalized in the infirmary of Medical Clinic of HULW/UFPB ( $n=140)$.

\begin{tabular}{ccc}
\hline Symptoms of delirium & $\boldsymbol{f}$ & \% \\
\hline Change in sleep-wake cycle & 23 & 16.4 \\
Difficulty focusing attention & 15 & 10.8 \\
Acute change in mental state & 9 & 6.4 \\
Change in level of consciousness & 14 & 10 \\
Memory impairment & 14 & 10 \\
Psychomotor retardation & 12 & 8.6 \\
Disorientation & 6 & 4.3 \\
Disorganized thinking & 5 & 3.6 \\
Change in perception & 2 & 1.4 \\
Psychomotor agitation & 1 & 0.7 \\
\hline
\end{tabular}

talization, but neither in the onset. Therefore, the incidence rate of delirium was $2.1 \%$ during hospitalization.

The variables ethnicity and origin were not correlated with the onset of delirium, but it was observed that patients who declared themselves inactive had a higher rate of development of delirium (10.3\%) than those who were active (2.5\%).

There was a significant association between age and delirium detected by the CAM $(p=0.03)$, with a higher frequency in older patients. Among the elderly, the prevalence of CAM positive was $10.8 \%$, while in patients younger than 60 years, this rate was $3.9 \%$. The average age of patients with delirium was 58.6 ( \pm 15.6) years, while that of patients who did not was $46.6( \pm 15.5)$ years and there was a statistical difference between the mean age of both groups ( $p=0.04$ ).

The duration of hospital stay did not differ between patients with delirium $(18.9 \pm 12.6$ days) than those who did not have this disorder (22.2 \pm 14.4 days). There was also no significant statistical correlation between the number of previous medical assistance and number of previous hospitalizations with delirium.

The variables history of head trauma, tuberculosis, alcohol consumption, previous episodes of delirium tremens, smoking, use of benzodiazepines, illicit drug use and prior history of depression did not significantly associated with positivity in the CAM. Among the medications used by patients and listed above, which showed statistically significant association with occurrence of delirium were antibiotics $(p=0.02)$, sympathomimetics $(p=0.03)$ and antihistamines $(p=0.004)$.

Immobilization in bed by mechanical restraint was significantly associated with the presence of delirium ( $p$ $=0.0001)$. However, it was found that the patients con- tained in the bed had higher median age than the younger.

Was statistically significant $(p=0.006)$ association between history of cognitive impairment and delirium. Patients who had chronic cognitive impairment showed delirium in $33.3 \%$ of cases, while $4.4 \%$ of those without this history reached the cutoff point of the CAM. Another variable that was associated with delirium was a history of psychiatric hospitalization ( $p=0.03$ ).

The occurrence of delirium in the early admission had a statistically significant association with hospital mortality $(p=0.03)$. It was found that $37.5 \%$ of patients who died during hospitalization had symptoms compatible with delirium at admission, higher than $11.4 \%$ in patients without that outcome.

\section{DISCUSSION}

The characterization of the sample of patients shows a predominance of middle-aged and elderly, corroborating previous research in general clinical care of LWUH [9]. The age of the patients in this study was diverse, although there was a predominance of middle-aged patients, one third of the sample of elderly.

The primary variable of this study was based on the application of the Confusion Assessment Method, which is an instrument designed to perform diagnosis of delirium, needing for this the presence of items one and two of the scale (acute onset and disorder of attention) and/or items three and four (disorganized thinking and altered level of consciousness) [6].

The prevalence of delirium found in this study was lower than the rates reported in the literature, but published studies focus mainly on the prevalence of delirium in elderly patients. Chavéz-Delgado et al. observed a prevalence of $38.3 \%$ and $11.7 \%$ incidence of delirium among elderly hospital patients [10]; patients who developed delirium were older than those who did not develop this disorder. In another study [11] at a university hospital (clinical and surgical infirmaries), the prevalence of delirium was $15 \%$ in the elderly, with an incidence of $26 \%$. Inouye et al. reported a prevalence of $11.8 \%$ in a cohort of hospitalized elderly [12].

Even in a study about the prevalence of delirium in a cohort of 133 hospitalized patients of different ages, there was a rate of $15 \%$, almost three times the proportion found in this study [13]. However, we must consider the heterogeneity of clinical presentation of patients with delirium.

However, it must be noted that prevalence rates vary greatly from one health service to another. Ouimet et al. [14] highlight this point, reporting that the prevalence of delirium in intensive care units, for example, varies between $11 \%$ and $80 \%$, finding in its own sample rate of 
31.8\%. It is assumed that the prevalence should also vary in the tertiary care services as that of population included in this study.

The incidence observed in this study was small, smaller than the initial prevalence, which may indicate the result of improvement of clinical and general conditions of patients, reducing the occurrence of the range of risk factors, like metabolic, hemodynamic factors, infectious diseases, electrolyte disturbance, among other factors that lead to delirium during a hospitalization.

It should be noted that, according to the CAM, a large number of patients with delirium had altered level of consciousness and change in sleep-wake cycle. Most patients showed disorganized thinking, but a smaller proportion showed disorientation, impairment of memory and psychomotor agitation. It called attention to the low prevalence of perceptual disturbances in the sample.

The clinical features of delirium are multiform and, to complicate the picture further, they vary rapidly with time. This variability and fluctuation is characteristic of delirium, and could also lead to diagnostic confusion. However, the appearance of lucid intervals in the clinical course of patients is an important observation, being diagnostic factor of delirium.

Moreover, the change of the framework in relation to the change in CAM score was small. These data are difficult to compare with the literature because of the complexity and multiplicity of variables involved in the development of delirium, in addition to the aforementioned clinical heterogeneity of the syndrome of delirium.

The use of the psychometric scale CAM for the search of delirium is associated with satisfactory validity and reliability. Although the usual clinical examination has shown good specificity (99\%) to detect the syndrome in elderly patients in an emergency department, the sensitivity was low (35\%) [15]. Furthermore, a study by Zou et al. showed superiority of CAM in relation to multiple clinical observations, with better sensitivity and specificity in the detection of delirium [16]. Thus, it is considered that the internal validity of the results was not the cause of the variability in the prevalence of the condition.

In relation to outcome, the most important was the relationship of delirium with in-hospital mortality. Was statistically significant relationship between CAM and mortality, which corroborates the literature that this syndrome is associated with increased mortality $[13,16]$.

The existence of basic medical illnesses, especially those that were mentioned, represents a major risk factor for the occurrence of delirium. These diseases are usually associated with the occurrence of delirium [17]. However, the most common etiology of delirium is multifactorial, involving predisposing and precipitating factors $[18,19]$.
The prior alcohol abuse is also a very important risk factor for the occurrence of delirium [20], but this association was not found in this work, nor the relationship with previous episodes of delirium tremens.

The drug prescription is implicated in up to $40 \%$ of cases of delirium and should be considered a predisposeing factor $[1,20]$. Virtually any drug can trigger delirium, but the main delirium-related drugs are benzodiazepines, anticholinergics and narcotic analgesics. In this study, was observed an association of delirium with antibiotics, which may represent a confounding factor, whereas infections underlying the use of these drugs were associated with delirium.

Inouye et al. report that the male gender is a pre- disposing factor for developing delirium, but in the pre- sent study the difference in the occurrence of delirium between males and females was not statistically signifycant, being $4.8 \%$ in men and $6.5 \%$ in women [21].

Individuals with previous brain injuries and old age are very vulnerable to developing delirium. In addition, certain conditions are associated with delirium: sleep deprivation, sensory loss (in particular, visual or auditory), immobilization, dehydration and malnutrition [19]. Pompei et al. reported the association between delirium and prior cognitive impairment [11]. It was observed in other study that $48.5 \%$ of patients who developed delirium had a history of sensory loss, which agrees with the results of this study [22].

Patients with delirium present, compared with patients in control groups, higher risk for mortality during hospitalization and after discharge [23]. In this sense, it must be noted that besides the epidemiological relevance of this work, there is an additional important contribution: the recognition of this disorder in routine of infirmary of medical clinic of the LWUH, because delirium is commonly under-diagnosed; approximately $70 \%$ of cases are not recognized by physicians [22], leading to an increase in mortality and morbidity. In this sense, in hospitals where high proportions of patients with delirium are treated, as a comorbidity of a somatic main diagnosis, should be included provisions for early detection and intervention in patients with this condition [24].

It is reported in the literature the relationship between delirium and longer hospital stay [11,22,25], but in the present study, there was no such association. However, the literature also shows works that there were no differences in hospital stay after an episode of delirium, such as Mittal et al., a cohort study of hospitalized patients [26].

Inouye et al. relate to five risk factors for delirium were identified: dementia, visual deficit, functional impairment, high comorbidity and use of mechanical restraint in bed [21]. This last association was found in this work, but the detention was more frequent in elderly 
patients who were the patients with higher rates of delirium, and therefore the restraint may have acted as an intervening variable in this relationship. The patients contained in the bed had higher median age than the younger ones, indicating the participation of the age variable in the relationship between immobilization in bed and positivity on the CAM. This association should be further studied so that accurate inferences can be made.

Considering the multifactorial model proposed by Inouye et al. for explanation of delirium, there is a complex relationship between a vulnerable patient with predisposing factors such as severe clinical disease, cognitive impairment, sensory deficits and precipitating factors, occurring during the hospitalization of the patient [18]. These aspects are highly prevalent in the clientele interned in internal medicine wards of LWUH and therefore, the syndrome of delirium should be considered in all patients attending the service, to facilitate recognition and to reduce morbidity and mortality in this population.

It concludes that the overall prevalence of delirium in the sample was $5.7 \%$, a rate lower than that found in other studies, and the incidence of delirium, $2.1 \%$, was small. The latter may have resulted from improvement of clinical conditions of patients and reducing precipitating factors of delirium because of the established treatment for the underlying disease. Was evident the importance of age as a consistent risk factor for developing delirium during hospitalization, besides the fact that patients had delirium on admission had a higher rate of hospital mortality.

Therefore, recognition of this disorder should be considered in routine of clinical wards of the LWUH, where patients are treated with multiple chronic illnesses, because delirium is most commonly under-diagnosed, leading to an increase in mortality rates. The diagnosis of delirium, a purely clinical act, based on patient observation and appreciation of the data provided by the family, should be systematically performed in patients in this context to reduce the under-diagnosis.

\section{REFERENCES}

[1] González, M., Pablo, J. and Valdés, M. (2003) Delirium: La confusión de los clínicos. La Revista Médica de Chile, 131, 1051-1060.

[2] Homsi, J. and Luong, D. (2007) Symptoms and survival in patients with advanced disease. Journal of Palliative Medicine, 10, 904-909. doi:10.1089/jpm.2007.0004

[3] Wacker, P., Nunes, P. V. and Forlenza, O. V. (2005) Delirium: Uma perspectiva histórica. Revista de Psiquiatria Clínica, 32, 97-103.

[4] Rudberg, M.A., Pompei, P., Foreman, M.D., Ross, R.E. and Cassel, C.K. (1997) The natural history of delirium in older hospitalized patients: A syndrome of heterogeneity. Age Aging, 26, 169-174. doi:10.1093/ageing/26.3.169

[5] Cole, M.G. and Primeau, F.J. (1996) Prognosis of delirium in elderly hospital patients. Canadian Medical Association Journal, 149, 41-46.

[6] Inouye, S.K., van Dick, C.H., Alessi, C.A., Balkin, S., Siegal, A.P. and Horwitz, M.I. (1990) Clarifying confusion: The confusion assessment method. A new method for detection of delirium. Annals of Internal Medicine, 113, 941-948.

[7] Organização Mundial da Saúde (1997) Classificação Estatística Internacional de Doenças e Problemas Relacionados à Saúde. Universidade de São Paulo, São Paulo.

[8] Fabri, R.M.A., Moreira, G.R. and Almeida, O.P. (2001) Validação e confiabilidade da versão em língua portuguesa do confusion assessment method (CAM) para a detecção de delirium no idoso. Arquivos de Neuro-Psiquiatria, 59, 175-179.

[9] Sousa-Muñoz, R.L., Maroja, J.L.S., Cunha, G.N., Trocolli, M.S. and Barbosa, J.A.D. (2005) Detecção clínica de consumo alcoólico em pacientes internados no hospitalescola da UFPB. Revista Brasileira de Medicina, 62, 387390.

[10] Chávez-Delgado, M.E., Virgen-Enciso, N., Peréz-Guzman, J., Celis-de-La-Rosa, A. and Castro-Castañeda, S. (2007) Detection of delirium in hospitalized elderly patients using the confusion assessment method. Revista Medica del Instituto Mexicano del Seguro Social, 45, 321-328.

[11] Pompei, P., Foreman, M., Ynouye, S.K. and Braund, V. (1994). Delirium in hospitalized older persons: outcomes and predictors. Journal of the American Geriatrics Society, 42, 809-815.

[12] Inouye, S.K. (2006) Delirium in older persons. The New England Journal of Medicine, 354, 1157-1165. doi:10.1056/NEJMra052321

[13] Thomas, R.I., Cameron, D.J. and Facs, M.C. (1988) A prospective study of delirium and prolonged hospital stay. Exploratory study. Archives of General Psychiatry, 45, 937-940. doi:10.1001/archpsyc.1988.01800340065009

[14] Ouimet, S., Kavanagh, B.P., Gottfried, S.B. and Skrobik, Y. (2006). Incidence, risk factors and consequences of ICU delirium. Intensive Care Medicine, 33, 66-73. doi:10.1007/s00134-006-0399-8

[15] Elie, M., Rousseau, F., Cole, M., Primeau, F., MacKusker, J. and Bellavance, F. (2000) Prevalence and detection of delirium in elderly emergency department patients. $\mathrm{Ca}$ nadian Medical Association Journal, 163, 977-981.

[16] Zou, Y. (1998) Detection and diagnosis of delirium in the elderly: Psychiatrist diagnosis, confusion assessment method, or consensus diagnosis? International Psychogeriatrics, 19, 303-308. doi:10.1017/S1041610298005390

[17] Fann, J.R. (2000. The epidemiology of delirium: A review of studies and methodological issues. Seminars in Clinical Neuropsychiatry, 5, 64-74.

[18] Inouye, S.K. (1999) Predisposing and precipitating factors for delirium in hospitalized older patients. Dementia 
and Geriatric Cognitive Disorders, 10, 393-400. doi:10.1159/000017177

[19] Inouye, S.K., Rushing, J.T., Foreman, M.D., Palmer, R. M. and Pompei, P. (1998) Does delirium contribute to poor hospital outcomes? A three-site epidemiologic study. Journal of General Internal Medicine, 340, 669-676.

[20] Conn, D.F. and Lieff, S. (2001) Diagnosing and managing delirium in the elderly. Canadian Family Physician, 47, 101-108.

[21] Inouye, S.K., Zhang, Y., Jones, R.N., Kiely, D.K., Yank, F. and Marcantonio, E.R. (2007) Delirium at discharge: Development and validation of a predictive model. Archives of Internal Medicine, 13, 1406-1413. doi:10.1001/archinte.167.13.1406

[22] Chavéz-Delgado, M.E., Virgen-Enciso, M., Perez-Guzmán, J., Celis-de-la-Rosa, A. and Castro-Castañeda, S. (2007) Detection of delirium in hospitalized elderly patients us- ing the confusion assessment method. Revista Medica del Instituto Mexicano del Seguro Social, 45, 321-328.

[23] Santos. F.S. (2005) Mecanismos fisiopatológicos do delirium. Revista de Psiquiatria Clinica, 32, 104-112. doi:10.1590/S0101-60832005000300002

[24] Stevens, L.E., De Moore, G.M. and Simpson, M. (1998) Delirium in hospital: does it increase length of stay? Australian and New Zealand Journal of Psychiatry, 32, 805808. doi:10.3109/00048679809073869

[25] O’Keefe, S.L. and Lavan, J. (1997) The prognostic significance of delirium in older patients. Journal of the American Geriatrics Society, 45, 174-178.

[26] Mittal, D., Majithia, D., Keneth, R. and Ruddy, J. (2006) Differences in characteristics and outcome of delirium as based on referral patterns. Psychosomatics, 47, 367-375. doi:10.1176/appi.psy.47.5.367 